\title{
Retraction: Research on the Simulation of Neural Networks and Semaphores
}

\author{
Haibo Zhu \\ Harbin University of Commerce, Harbin, China \\ haibo26@yahoo.cn
}

Several conference proceedings have been infiltrated by fake submissions generated by the SCIgen computer program. Due to the fictional content the chapter "Research on the Simulation of Neural Networks and Semaphores" by "Haibo Zhu" has been retracted by the publisher. Measures are being taken to avoid similar breaches in the future. 